\title{
A competitive cell-permeable peptide impairs Nme-1 (NDPK-A) and Prune-1 interaction: therapeutic applications in cancer
}

\author{
Veronica Ferrucci ${ }^{1,2,3} \cdot$ Francesco Paolo Pennino ${ }^{1,2} \cdot$ Roberto Siciliano $^{2} \cdot$ Fatemeh Asadzadeh $^{2} \cdot$ Massimo Zollo $^{1,2,3}$
}

Received: 19 June 2017 / Revised: 15 November 2017 / Accepted: 22 November 2017 / Published online: 15 February 2018

(c) United States \& Canadian Academy of Pathology 2018

\begin{abstract}
The understanding of protein-protein interactions is crucial in order to generate a second level of functional genomic analysis in human disease. Within a cellular microenvironment, protein-protein interactions generate new functions that can be defined by single or multiple modes of protein interactions. We outline here the clinical importance of targeting of the Nme-1 (NDPK-A)-Prune-1 protein complex in cancer, where an imbalance in the formation of this protein-protein complex can result in inhibition of tumor progression. We discuss here recent functional data using a small synthetic competitive cellpermeable peptide (CPP) that has shown therapeutic efficacy for impairing formation of the Nme-1-Prune-1 protein complex in mouse preclinical xenograft tumor models (e.g., breast, prostate, colon, and neuroblastoma). We thus believe that further discoveries in the near future related to the identification of new protein-protein interactions will have great impact on the development of new therapeutic strategies against various cancers.
\end{abstract}

\section{Therapeutic potential of CPP}

In human cells, protein-protein interactions (PPIs) have been estimated to range from 14,000 binary interactions to over 650,000 multi-component interactions, and the detailed definition of PPI networks has provided new insight into many protein functions [1]. Following dysregulation of several protein complexes involved in PPIs, inhibition of specific complexes has been shown to represent an attractive therapeutic strategy for several pathologic conditions, including cancers (see databases of Kyoto Encyclopedia of Genes and Genomes [KEGG] of cancer; http://www.genome.jp/kegg/disease/cancer.html).

To date, improvements in the technologies aimed at the detection of protein structures have produced different

Massimo Zollo

massimo.zollo@unina.it

1 Dipartimento di Medicina Molecolare e Biotecnologie Mediche, Università degli Studi di Napoli Federico II, Via Sergio Pansini 5, 80131 Naples, Italy

2 CEINGE Biotecnologie Avanzate, Via Gaetano Salvatore 486, Naples, Italy

3 European School of Molecular Medicine (SEMM), University of Milan, Milan, Italy synthetic PPI inhibitors that can target specific PPIs and affect their downstream signaling cascades [2, 3]. Indeed, PPIs have been shown to have roles in the regulation of fundamental intracellular processes, including the functions of cell-cycle proteins that are crucial for cell division. An example of the importance of these protein complexes can be seen for the cyclin-dependent kinases family (i.e., cdk1-8) and their corresponding cyclin proteins (cyclinAI). Overall, these proteins are finely regulated by PPIs during the cell cycle, in mitosis. The cdk proteins alone do not show any kinase activity, but instead they acquire the ability to phosphorylate intracellular proteins when in association with specific cyclin partners. These interactions, in turn, generate protein complexes with additional functions that can drive the cell cycle, and then promote cell division [4].

PPIs are also of importance in the regulation of cell apoptosis. The proteins involved here thus include those that are active during protein shuttling from the nucleus to the cytoplasm. One of the most important coordinators of cell apoptosis is the tumor suppressor protein TP53, whereby its interactions with various binding partners control a spectrum of intracellular actions. In this context, the E3-ubiquitin-ligase $\mathrm{Mdm} 2$ (murine double minute 2; human homolog hdm2) has been shown to be essential for the regulation of growth-suppressive TP53 functions through modulation of TP53 polyubiquitination and 
proteosomal degradation [5]. In turn, TP53 can activate $\mathrm{Mdm} 2$ at the transcriptional level, thus promoting an autoregulatory feedback loop [6]. In addition, the binding of TP53 to the anti-apoptotic $\mathrm{Bcl} 2$ family members prevents their interactions with pro-apoptotic proteins (e.g., BCL2 associated $\mathrm{X}$, or Bax; alternatively known as BCL2 antagonist/killer 1, or Bak), which results in inhibition of their pro-apoptotic effects in mitochondria [7].

Furthermore, several more PPIs that are directly involved in tumor progression have been described, which include TP53 and Mdm2 in tumors that show overexpression of Mdm2, thus resulting in reduction of the anti-neoplastic activity of TP53. Here, recent studies on characterization of the binding sites between Mdm2 and TP53 have allowed mimetic small molecules to be developed, which are also known as "Nutlins" (e.g., cis-imidazoline analogs) [8]. Additional analogs have been described that can bind Mdm2 within the TP53-binding pocket in a competitive manner, thus further inhibiting the complex formation and enhancing the TP53 signaling pathway [8].

Here we describe another intracellular cytoplasmic PPI that results in a physical interaction between the Nme-1 (also known as NDPK-A) and Prune-1 proteins. This complex has been shown to be associated with cancer aggressiveness and poor patient prognosis. The Nme1-Prune-1 interaction complex has been reported to induce cell migratory properties and metastatic dissemination in several tumor malignancies [9]. Here, we review studies related to the formation of the Nme-1-Prune-1 complex in vitro, and in particular to the development and use of a small synthetic competitive cell-permeable peptide (CPP) that illustrates the therapeutic benefits of impairing the interactions between the Nme-1 and Prune-1 proteins in adult and pediatric solid tumors (e.g., breast, prostate and colorectal tumors, and neuroblastoma) [10, 11].

Genetic interactions between Nme-1 and Prune-1 were first reported in Drosophila melanogaster, where both homozygous and hemizygous Prune (Pn) mutants that are responsible for the brownish-purple "prune" eye color were shown to be lethal in the presence of just a single mutation (i.e., P97S substitution) in the Nme-1 protein (the NDPK-A orthologue; alternatively known as $a w d$, abnormal wing disks gene). These double mutants die at the second to third larval instar stage due to abnormal development of mesoderm and central-nervous-system-like cells [12, 13]. Interestingly, this P97S awd mutant can still bind to the Prune-1 protein, while the interaction between Prune-1 and awd is impaired in the presence of the S120G awd mutant protein [14]. The lethal interaction was hypothesized be due to neomorphic mutations that caused alterations during the GTP and GDP switch mediated by the awd protein in its regulation of intracellular GTPases and GTPase-activating proteins [15].
Of particular importance, the first evidence in mammals of Nme-1 and Nme-2 (also known as NDPK-B) and Prune1 as co-expressed proteins arose from embryonic tissues within the central nervous system [16]. Indeed, both of the NM23-M1 and PRUNE-M1 proteins (i.e., murine homologs of human Nme-1 and Prune-1, respectively) showed the same spatio-temporal expression patterns in several regions of the developing nervous system (from E10.5 to adulthood). This thus suggested fundamental functions for NM23-M1 and PRUNE-M1 during the cell proliferation, migration and differentiation processes that occur in the development of the brain and cerebellum [16]. An additional role of the complex between Nme-X1 and Prune-X1 (i.e., Xenopus homolog proteins) was reported for Müller gliogenesis during Xenopus retinal development, which thus indicated an important role for Nme-1 and Prune-1 during the development of the retina and in eye morphogenesis [17]. Nme-1 was first identified as having an antimetastatic activity in human through differential colony hybridization studies that were performed on different cell lines derived from a murine melanoma (K-1735 cells) that showed varying metastatic potential [18]. The gene here was originally named as "Non-metastatic 23" (NM23), and then Nm23-M1 (i.e., murine homolog gene) after the discovery of a second $\mathrm{Nm} 23$ gene that was defined as Nm23M2 [19]. Later, the human counterparts Nm23-H1 and Nm23-H2 were identified through cDNA library screening in human fibroblasts, with these genes also referred as NDPK-A and NDPK-B (i.e., NME1, NME2, respectively) [20].

Of note, Nme-1 was reported as being overexpressed in several tumorigenic cell lines, which included melanoma, breast and oral squamous cell carcinomas and lymphomas, and to reduce cell motility in vitro and inhibit metastatic dissemination in vivo [18, 21-23]. Notwithstanding these reports, high Nme-1 protein levels have also been positively correlated to poor patient prognosis, with their expression levels associated with low overall survival for patients affected by osteosarcoma and hematological tumors [24, 25]. These different behaviors of Nme-1 in several tumors can be explained in part by its multiple functions (i.e., nucleotide diphosphate kinase: NDPK [26] histidine protein kinase [27, 28]; 3'-5' exonuclease activity [29], and also by its potential to bind to different interactor proteins that are involved in several signaling pathways, including G-protein complexes and their functional activation, mitogenactivated protein kinase, and the transforming growth factor- $\beta$ signaling cascade, and its association with cytoskeleton proteins (e.g., $\beta$-tubulin) [30].

Prune-1 belongs to the phosphoesterase $\mathrm{DHH}$ protein family and it has also been shown to be positively regulated by Nme-1 [9]. Of importance, Prune-1 has an exopolyphosphatase activity (PPX/PPAse) that shows higher 
A

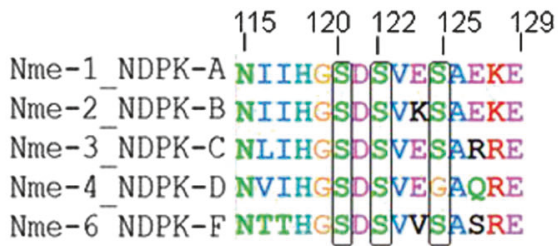

B

human
kangaroo
guinea
mouse
rabbit
lemur
orango
dog
cat
fugu
cow
sheep
xenopus
zebrafish

Prune-1

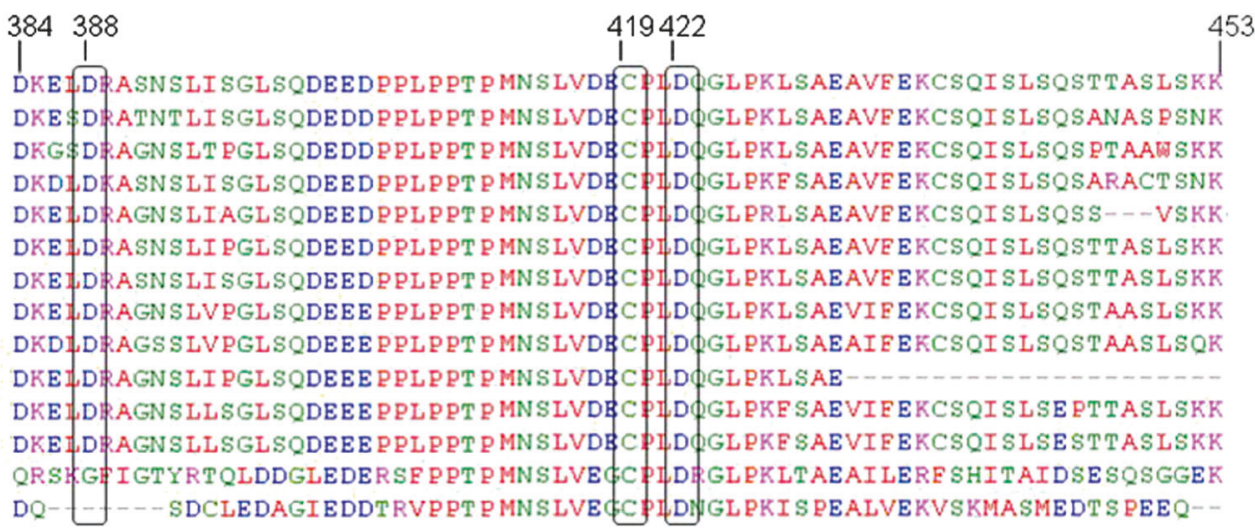

Fig. 1 (A) Sequence alignment of the region of the human Nme-1, -2, $-3,-4$ and -6 proteins (also known as NDPK-A, -B, -C, -D,-F) that are mainly involved in the interactions with the Prune-1 protein (residues 115 to 129), illustrating their conservation with a high degree of identity and homology across their isoforms. Within this carboxylterminus region of these Nme proteins, serines 120, 122 and 125 are also conserved (black boxes). These residues show high probability scores for phosphorylation mediated by intracellular casein kinases I and II, and their phosphorylation is necessary for the binding of Nme-1 and Nme-2 (NDPK-A, -B) with Prune-1. (B) Alignament of the sequence region of the carboxyl-terminus domain of Prune-1 (residues 384-453), which is involved in the binding to Nme-1, shows the

affinity for short-chain over long-chain inorganic polyphosphates [31]. This Prune-1 PPX/PPase activity was shown to be negatively influenced through Prune-1 binding with Nme-1, thus further confirming that the Nme-1-Prune-1 complex might acquire a different function in terms of influencing the pool of inorganic polyphosphate levels in cellular compartments [31].

To date, literature data that highlight the association between dysregulated Prune-1 activity and tumor progression have been described for patients with highly metastatic breast [32], gastric, esophageal [33], non small-cell lung [79], and colorectal [34] cancers. The binding of Prune-1 to Nme-1 and Nme-2 was also confirmed using coimmunoprecipitation assays in human breast cancer cells (i.e., MDA-MB-435 cells) [35]. In mammalian cells, alterations in the equilibrium of the Nme-1-Prune-1 interaction complex are responsible for the switch to pathological conditions (i.e., cancer) through effects on specificrelated protein networks $[36,37]$. Thus, the Nme-1 and conservation through evolution across different vertebrates with a high degree of sequence identity and homology. The amino-acid residues that are mainly responsible for the interaction between Prune-1 and Nme-1 are shown in black boxes (i.e., D388, C419, D422). These three residues lie within the proline-rich motif of Prune-1 and are shared between species (vertebrates, chordates), excluding Xenopus laevis and Zebrafish (with potential divergency in tetrapods), but including the Fugu rubripes (Fugu fish) genome. The region from 420 to the end of Prune-1 (amino acid 453) is responsible for its dimerisation, which has been reported to interact with the hexameric form of Nme-1 (NDPK-A) [41]. These sequence alignaments were realized using the ClustalX 2.1 software.

Prune-1 protein interaction might help to explain the dual role of Nme-1 in its actions as a metastasis-suppressing or metastasis-promoting protein, as first described for the Nme proteins [30].

Studies on the regions of interaction between Nme-1 and Prune-1 have finely mapped the region of their interaction through affinity chromatography approaches and Western blotting analyzes in human breast cancer cell lines. These studies indicated that the carboxyl-terminus (C-term) domains of both of these proteins are responsible for their interaction [35]. Then, a three-dimensional model of the C-term domain of Prune-1 (C-term-Prune-1) was obtained through nuclear magnetic resonance spectroscopy studies combined with homology modeling and molecular dynamics approaches. This tertiary structure of C-term-Prune-1 provided further insight into the aminoacid regions that are involved in the interaction between Prune-1 and Nme-1 and Nme-2 [10] . Interestingly, these data reported the presence of two globular domains in the 
A
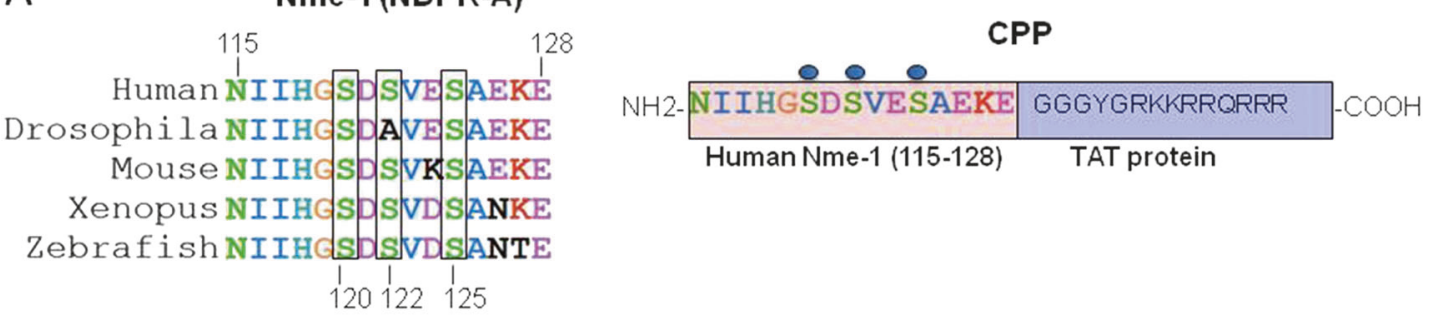

B

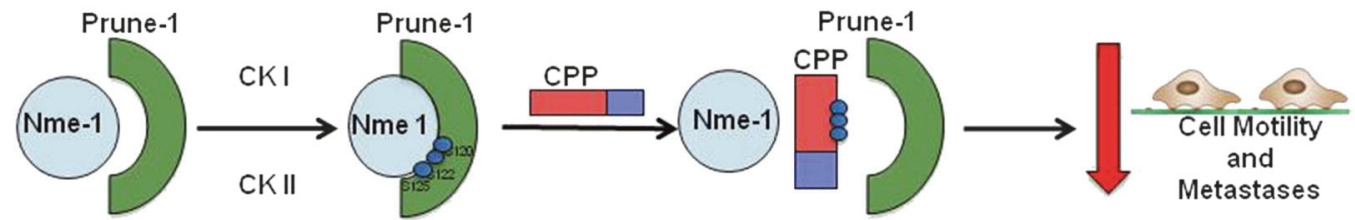

Fig. 2 (A) Left: Sequence region of the carboxyl-terminus domain of the Nme-1 (NDPK-A) protein that is responsible for its interaction with Prune-1 (residues 115-128). This is higly conserved across different species (e.g., Homo sapiens, Drosophila melanogaster, Mus musculus, Xenopus leavis, Zebrafish) with a high degree of homology and identity. Right:The amino acids of the carboxyl-terminus domain of the Nme-1 protein (N115-E128; red box) used for the development of the competitive cell-permeable peptide (CPP) that was designed to impair the Nme-1-Prune-1 interaction complex. The selected sequence contains the three serine residues that are important for phosphorylation mediated by Casein Kinases I and II (i.e., S120, S122, S125). This phosphorylation is necessary for the interaction of Nme-1 with Prune1. This sequence was then fused to the trans-activating protein region

$\mathrm{N}$-terminus region (DHH, DHHA2) that are responsible for the Prune-1 enzymatic activities [10]. An intrisically disordered domain was then identified in Prune-1 that begins at amino-acid residue 371 and contains two stretches with a propensity for helix formation, and a small globular region that mediates Prune-1 binding to Nme-1 and Nme-2 [10]. Together with site-direct mutagenesis approaches, these mapping data identified the D388 and D422 residues of Prune- 1 as the most conserved amino acids of the C-term-Prune-1 region that is involved in Prune-1 binding to Nme-1. In summary here, through alignments performed with ClustalX 2.1, these regions of interaction between the Nme-1, Nme-2, Nme-3, Nme-4, and Nme-6 (NDPK-A, NDPK-B, NDPK-C, NDPK-D, NDPK-F) and Prune-1 proteins are highly conserved in these amino-acid sequences in human (Fig. 1a).

The Prune-1 region of interaction with Nme-1 was then also shown to retain a region with a high degree of similarity across different species (Fig. 1b). These data further implied that this functional interaction that leads to the complex formation must have a fundamental role in cell physiology (e.g., potentially regulating cell migration) as it has been maintained in all vertebrates through natural selection. Indeed, the D388A and D422A Prune-1 mutant proteins (where the D388 and D422 residues of Prune-1 were mutated to alanine) only weakly interacted with Nme- of the human immunodeficiency virus (blue box) for the generation of the CPP with adenovirus carrying vector of type V. The sequence alignament was realized with the ClustalX 2.1 software. (B) Representative model showing the intracellular mechanism of action of CPP. This synthetic CPP can compete with the intracellular Nme-1 (NDPKA) protein for phosphorylation on the S120, S122 and S125 residues mediated by Casein Kinase I and II, thus reducing Nme-1 phosphorylation and impairing Nme-1 binding to the Prune-1 protein. The treatment with CPP thus impairs the Nme-1-Prune-1 interaction and results in reduction of tumour progression and metastatic dissemination for several solid tumours in animal models of cancer (i.e., breast, prostate and colorectal cancer, and neuroblastoma).

1 and did not induce any pro-migratory phenotype when overexpressed in mammalian cells (e.g., HEK-293T cells) [10]. These data are of particular importance for the correlation of Nme-1-Prune-1 protein complex formation with a pro-migratory phenotype, and they further strength the functional consequences, considering that this is a function that would have been positively selected for through evolution.

Furthermore, nuclear magnetic resonance spectroscopy has allowed the Nme-1 and Prune-1 interaction to also be observed in different human cells (e.g., HEK-293T, SHSY5Y cells), thus confirming the formation of a high molecular weight complex between Nme-1 and Prune-1 directly in human cell lysates [38]. Thus, single-point mutations of Nme-1 that are known to alter its biochemical activity (e.g., P96S, S120G) were shown to negatively affect the Nme-1 interaction with Prune-1, and also cell motility. This thus confirmed that the loss of the activity of Nme-1 mutant proteins (e.g., NDPK-A-P96S, NDPK-AS120G mutants) was mainly due to their lack of binding to Prune-1 [38]. Once again, the Nme-1-Prune-1 protein complex was shown to have a fundamental role in these neuroblastoma tumorigenic cells, thus further confirming its role in cancer progression.

Through bioinformatic analyzes of the $S(p)-x-x-S$ consensus sequence for casein kinase I and II (CKI, CKII), the 
regions of Nme-1 and Nme-2 from their serine 120 (S120) to serine 125 (S125) residues were shown to contain candidates with high probability scores for phosphorylation mediated by CKI and CKII, with a perfect match within this amino-acid region [35, 39]. Among the different isoforms of $\mathrm{CKI}, \mathrm{CKI} \varepsilon$ was reported as the most efficient for phosphorylation of Nme-1 using in-vitro kinase assays [35]. These residues (S120, S122, S125) appeared to be responsible for the intracellular interaction of Nme-1 with Prune-1, and indeed, single point mutations in these amino acid positions of Nme-1 were independently shown to affect its interaction with the human Prune-1 protein. Moreover, invitro studies demonstrated that the Nme-1-Prune-1 interaction complex was only seen when Nme-1 had been previously phosphorylated by CKI [35]. These findings suggested that CK-mediated phosphorylation of Nme-1 is a physiological condition that is necessary for this PPI within cells in a particular cellular environment.

Furthermore, phosphorylation of Nme-1 by CKI influences its oligomerisation status, with the recombinant Nme1 protein showing an increase in its hexameric structure upon CKI phosphorylation (53.6\% hexamer, $42.2 \%$ trimer, and $4.1 \%$ dimer). Further, addition of the recombinant Prune-1 protein during CK-induced phosphorylation of Nme-1 showed that Prune-1 dimers can bind to the hexameric structure of Nme-1 [40]. Altogether, these data clarified the interaction regions of both Nme-1 and Prune-1 at the molecular level. This, thus confirmed the formation of a high-molecular-weight complex that included six Nme-1 molecules linked to two Prune-1 protein monomers.

At this time, a competitive cell-permeable peptide (CPP) that mimics the minimal region of interaction of Nme- 1 and Nme-2 (i.e., amino acids 115-128) was designed to impair the formation of the Nme-1-Prune-1 complex [35, 38]. Clinical shift mapping was applied to dissect out the interactions of Nme-1 and CPP with C-term-Prune-1. Here, the interaction surface, seen as a smaller amino-acid region within CPP, preserved most of the amino acids that belong to the residues 387-396 region and a smaller part of the Cterm globular region [10]. Then, in vitro in cells, CPP was shown to reduce the phosphorylation of Nme-1 mediated by CKI and CKII, and to subsequently impair the formation of the Nme-1-Prune-1 complex [35]. For this purpose, the amino-acid sequences for the Nme-1-Prune-1 interaction were fused to the trans-activating protein of the cellpenetrating region of human immunodeficiency virus, and this was used in vitro to confirm its activity within the use of an adenoviral-carrying vector type V (see Fig. 2a) that can synthesise this peptide in vivo once it has infected tumorigenic cells that represented different tumor types. The following paragraphs describe the use of this adenovirus for therapy in vitro and then in in-vivo preclinical studies in mouse tumor models.
Breast cancer is the most common malignant disease in women. Among the major subtypes of breast cancer, basallike breast cancer (also known as triple-negative breast cancer) is the most aggressive and the least sensitive to standard chemoradiation regimens currently used in clinics [41]. Overexpression of Nme-1 has been reported in several metastatic cell lines, and Nme-1 has been described as a metastasis suppressor in breast cancer [42]. In this context, Nme-1 has been reported to reduce cell motility when overexpressed in breast cancer cells [43]. Conversely, high tissue levels of Nme-1 expression have been shown in patients with breast cancer, and statistically positive associations of high Nme-1 expression and poor overall survival and relapse-free survival have also been reported [44]. These opposing roles for Nme-1 in metastatic breast cancer can be partially explained by taking into account the extracellular Nme-1 protein that was identified [45, 46]. Interestingly, the Awd (orthologue of Nme-1/ NDPK-A) protein was also detected in the extracellular environment in the D. melanogaster model [47]. Indeed, both of the Nme-1 and Nme-2 proteins have been found in culture medium supernatants collected from breast cancer cell lines [46]. Moreover, these same proteins were also found in the sera from patients with breast cancer, with higher levels in those women with metastases, thus suggesting an extracellular role with a positive correlation to metastatic progression in breast cancer [46].

Furthermore, secreted extracellular Nme-1 and Nme-2 have also been proposed to have roles in the promotion of angiogenesis in breast cancer. Indeed, several breast cancer cell lines have been shown to induce endothelial cell tubulogenesis through secretion of both Nme-1 and Nme-2 [48], thus resulting in endothelial cell growth and migration through activation of vascular endothelial growth factor receptor type 2 and extracellular signal-regulated kinase [49], or by modulation of the levels of nucleotides in the tumor vasculature (e.g., increased ATP levels), to promote the release of vasoactive factors with angiogenic activities. On the other hand, this metastatic malignant breast cancer behavior has also been positively associated with clonal selection amplification of the 1q21-q22 chromosomal region. This common aberration occurs more frequently in metastatic lesions than in primary lesions, and it is associated with short patient overall survival $[50,51]$ and chemotherapy resistance [52, 53]. The PRUNE-1 gene is located on 1q21.3, which is a region that is also amplified in advanced breast cancers [54]. Of interest, the overexpression of Prune-1 was shown for tumors that were also characterized by higher Nme-1 expression levels [54]. These data suggest that in this set of clonal selected tumors with amplification of the 1q21-22 region and with high Nme-1 expression levels, the increased Prune-1 levels can enhance tumor progression and metastatic dissemination. 
Tissue multiple arrays on primary tumor tissues derived from patients with metastatic breast cancer then confirmed this hypothesis [9]. Later, the distribution of Prune-1 was examined in a larger and better-characterized tissue cohort of invasive breast cancers. Here, the immunoistochemical analyzes revealed that the strong cytoplasmic immunoreactivity for Prune-1 observed in the majority of the breast cancer tumors tested (54\%) was significantly correlated with advanced nodal status (N2-N3 cases) and positive lymph-node status $(P=0.017)$, and with distal metastases $(P=0.029)$. Overall, the potential application of Prune-1 as a marker for identification of the subsets of patients with aggressive metastatic breast cancer was defined [32].

The potential to reduce the PPI complex formation through inhibition of the CK-mediated phosphorylation of Nme-1 (which is necessary for binding to Prune-1) was investigated using IC261 (ATP-competitive inhibitor, selective for $\mathrm{CKI} \delta$ and $\mathrm{CKI} \varepsilon$ [55]) in breast cancer cell lines. These data suggested that in a breast cancer cell model, this inhibition of phosphorylation of Nme-1 by IC261 can impair the formation of the Nme-1-Prune-1 complex, decrease the Prune-1 PDE enzymatic activity, and reduce cell migration [35]. Furthermore, and most importantly, this occurred with greater specificity in relation to serine inhibition of phosphorylation. Nonetheless, doubts were raised about the IC261 specificity of action against CKI $/ \varepsilon$ inhibition, as IC261 was also demonstrated to alter microtubule depolymerization [56]. Then the CPP was developed that mimicked the region of Nme-1 that is involved in the interaction with Prune-1, including Ser120, Ser122, and Ser125. This peptide was used in similar experiments, and it showed similar efficacy to IC261 [35].

Furthermore, therapeutic properties of CPP were identified in vitro using breast cancer cell lines and cell proliferation assays [11]. Indeed, block of the Nme-1-Prune-1 interaction using CPP reduced proliferation of breast cancer cells (i.e., MCF7, MDA-MB-231 cell lines), as measured using cell index proliferation assays. Of interest, CPP also inhibited the AKT/ mTOR and NF-kB signaling pathways in these treated breast cancer cells [11].

In prostate cancer [57], a prognostic role for Nme-1 has been defined [58]. We reported on a study in a large cohort where the analysis showed that Nme-1 is a negative prognostic factor for prediction of patient outcome [59]. As the Nme-1-Prune-1 interaction has been directly correlated to migratory properties of several tumorigenic cells, the potential for CPP to impair this complex formation was also investigated in the PC3 prostate cancer metastatic cell line [11]. Expression of CPP in PC3 cells was obtained using adenoviral particles that provided high efficiency of infection, and the CPP block of the binding between Nme-1 and Prune-1 was assessed in these cells using an affinity chromatography approach. Furthermore, CPP was also shown to impair cell proliferation and induce cell apoptosis in these PC3 cells (assayed as cell index of proliferation on a realtime system, and as caspase 3 activity assays, respectively). Of importance, the inhibition of PC3 cell migration by CPP was also determined through monitoring cell migration in real time using the xCELLigence RTCA DP system. These data showed that in vitro, CPP-treated PC3 cells had reduced migratory properties compared to control cells (infected with empty adenoviral particles) [11]. These data suggested a direct correlation between the Nme-1-Prune-1 interaction complex and enhancement of cell motility also in prostate cancer metastatic cell lines.

The potential for CPP inhibition of metastases arising from prostate cancer was then evaluated in vivo using an orthotopic murine xenograft model of human PC3-Luc cells (i.e., PC3 cells stable for firefly luciferase expression) injected into the dorsal prostate of athymic nude mice [11]. This xenograft metastatic model of prostate cancer had been previously reported to resemble the metastatic behavior of prostate cancer cells in humans with regard to the development of metastases [60]. Thus the potential antimetastatic effects of CPP were evaluated by delivery of adenoviral particles carrying the sequence that encoded CPP, through tail-vein injections in mice 1 month after prostate cancer surgical implantation. Two weeks later, the tumor-bearing mice were sacrificed and a decrease in tumor growth was shown in the CPP-treated mice. Most importantly, the lungs, liver, and femurs from each mouse were excised and analyzed using ex-vivo bioluminescence imaging, with suppression of macro-metastases seen for the CPP-treated group of mice. All of the control tumor-bearing mice showed macro-metastases in the lungs, liver or femur. In contrast, among the CPP-treated tumor-bearing mice, only one (of four) showed macro-metastases in the lungs, while none of them showed metastases in the liver or femur. This thus showed that CPP can be effective ffor inhibition of metastatic dissemination in prostate-cancer-bearing mice after a single dose of adenoviral particles administration [11].

Additionally and most importantly, the haematological status in the CPP-treated mice did not show any variations for white-blood cell, platelet and erythrocyte total cell numbers, and there was an absence of kidney and liver failure, as measured by creatinine and alanine aminotransferase levels. We also investigated whether CPP induced apoptosis in non-tumoral cells, using non-tumoral human embryonic kidney cells (i.e., HEK-293T cells), where CPP did not activate caspase 3. Altogether, we confirmed that there were no signs of toxicity of CPP in normal proliferating cells. This indicates further that the pro-apoptotic effects of CPP occur only in tumorigenic cells, thus reinforcing its therapeutic future applications in metastatic prostate cancer treatment [11]. 
In colorectal cancers that develop liver metastases, the role of Nme-1 in colorectal carcinoma still remains controversial, and its cellular mechanisms in terms of the metastatic phenotype are not yet fully understood [61]. The potential association of Nme-1 with liver metastases and survival in patients with colorectal carcinoma was investigated by performing multiple arrays of primary tissues [62]. Nme-1 expression was significantly higher in the colorectal carcinoma tissues than in the adjacent non-neoplastic mucosa $(P<0.0001)$, but it did not correlate with the presence of liver metastases or patient overall survival [62].

Nme-1 has also been reported to have a significant role in vitro in the reduction of migration of colon cancer cells (i.e., HT29 cells), and in vivo it was shown to suppress liver metastases, using xenograft mouse models [63]. Here, the group of mice injected with Nme-1-overexpressing cells showed lower liver weights, with fewer metastatic foci compared to the control mouse group [63].

The role of Prune-1 was also investigated in colorectal cancer liver metastases, both in vitro and in vivo. To this end, Prune-1 expression increased cell migration in vitro using colorectal cancer cell lines that showed high and low expression levels of their endogenous Prune-1: SW480 and HCT116 cells, respectively [34]. The Prune1-knocked-down SW480 (sh-Prune-1-SW480) cells and Prune-1-overexpressing HCT116 cells were assayed for cell migration in vitro. These data indicated the pro-migratory phenotype ascribed to the expression of Prune-1. Of importance here, the sh-Prune-1-SW480 cells also showed downregulation of mesenchymal markers (i.e., N-cadherin, vimentin), and a corresponding upregulation of epithelial markers (i.e., E-cadherin, Zo-1). These data suggested that Prune-1 expression is associated with epithelial-tomesenchymal transition, which is known to be crucial for metastasizing to distant organs. These data were also confirmed later in an in-vivo assay where sh-Prune-1-SW480luc cells (i.e., stably overexpressing the Firefly luciferase gene) were injected into the portal vein of NOG/Jic mice. The luciferase activity in the mice injected with sh-Prune-1SW480 was significantly lower in comparison with those injected with 'scrambled'-SW480 cells. Thus, Prune-1 was reported to promote liver metastases from colorectal cells also in vivo, mainly due to the activation of epithelial-tomesenchymal transition [34].

We additionally tested the anti-tumorigenic potential of CPP in colorectal cancer cells in vitro using an adenovirus delivery system [11]. For this purpose, both HT29 and SW480 cells were treated with CPP (delivered via the adenoviral technology), and cell proliferation was determined using cell index real-time proliferation assays. These data showed decreased proliferation rates for the CPPtreated cells compared to the control cells [11]. In addition, there was a significant increase in caspase 3 activity in the
CPP-treated cells, which thus suggested that the antiproliferative effects mediated by CPP are also due to activation of apoptosis in the treated cells [11]. Furthermore, CPP also showed decreased phosphorylated Nme-1 and inhibited the AKT and NF- $\mathrm{KB}$ pathways in both the HT29 and SW480 treated cells, thus definitively showing that the anti-proliferative effects of CPP arise from Nme1-Prune-1 complex regulation of the signaling cascades of metastatic colorectal cancer [11]. These data support the use of CPP for the treatment of animal models of colorectal cancer and metastasis formation, to confirm its efficacy in vivo.

Neuroblastoma is the most common embryonal malignancy of the sympathetic nervous system of early childhood [64]. Here, a single point mutation of Nme-1 that results in a Ser120-Gly substitution has been reported for $21 \%$ of patients with advanced neuroblastoma, with gain of chromosomal segment 17q21 also seen. The association of these Nme-1 aberrations with the high metastatic potential of human neuroblastoma strongly suggests that Nme-1 functions as a metastasis promoter protein [65].

The literature data indicate that overexpression and S120G mutation of Nme-1 promote neuroblastoma metastases by preventing neuronal differentiation and increasing neuroblastoma colonization and cell survival, with Nme-1 considered as a potential marker for prediction of clinical outcome of patients with neuroblastoma [66]. Furthermore, examination of the serum Nme-1 protein levels in 217 patients with neuroblastoma revealed significantly higher expression levels in patients with neuroblastoma than in control children. However, in these patients, high serum Nme-1 levels ( $\geq 250 \mathrm{ng} / \mathrm{mL}$ ) were positively associated with $\mathrm{N}$-myc amplification and negatively correlated with poor overall survival. These data are of importance, because they highlight the contribution of the serum Nme-1 protein levels to the predictions of clinical outcome in children affected by neuroblastoma [67].

We investigated the role of Nme-1-Prune-1 protein complex formation in neuroblastoma tumor progression and metastases [10]. Furthermore, the use of CPP with adenovirus delivery was investigated in neuroblastoma cells, to determine its therapeutic properties in vitro. When the expression of CPP was driven in neuroblastoma cells, impairment of the binding between Nme-1 and Prune-1 was seen, with decreased levels of phosphorylated Nme-1. As a consequence, a reduction in cell proliferation and induction of apoptosis were also reported (assessed by cell proliferation assays and caspase 3 activity measurements, respectively) [10], [11]. Most importantly, CPP also inhibited cellular motility in vitro in two different neuroblastoma cell lines (i.e., SH-SY5Y, SHEP cells). The anti-tumorigenic actions of CPP were also investigated in vivo here, using heterotopic xenograft mice injected with SH-SY5Y-Luc 
cells (i.e., that expressed the Firefly luciferase gene) previously infected with the adenoviral particles carrying CPP or the control (empty adenoviral particles; mock) using invivo biolumuniscence imaging technology. The mice that received CPP-treated SH-SY5Y-Luc cells showed decreased tumor burden compared to the control group. Interestingly, the tumors analyzed from the mice under the active treatment showed immunoreactivity staining for the neuronal marker Tuj1 (i.e., neuron-specific class III $\beta$ tubulin), which is indicative of neuronal differentiation processes, and caspase- 3 activation.

Altogether, these data demonstrated the therapeutic benefits of the use of CPP in neuroblastoma, where high expression of Nme-1 has been identified, together with Prune-1 expression. These findings are relevant for the potential treatment of pediatric neuroblastoma characterized by chromosome $17 \mathrm{q}$ gain and overexpression of Nme-1 [10].

\section{Future perspectives}

Genetic interactions between Nme-1 and Prune-1 D. melanogaster orthologue proteins (NDPK-A; AWD; Killer of Prune) where described by Biggs et al. [68]. Homozygous and hemizygous Prune-1 mutants were shown to be lethal in the presence of the P97S mutation in the awd protein, the Nme-1 orthologue. This genetic interaction has been conserved at the protein level throughout evolution, mainly in vertebrates, including Mus musculus, Danio rerio, and Xenopus laevis [14].

Efforts have been put into the mapping of the amino acids responsible for the Nme-1-Prune-1 interaction through nuclear magnetic resonance spectroscopy approaches, aimed at inhibition of this PPI within tumorigenic cells. Since then, phosphorylation of Nme-1 (on Ser120, Ser122, and Ser125) was shown to be mediated by consensus region phosphorylation by CKI and CKII [35]. Thus, targeting these phosphorylation events might represent a new therapeutic strategy to impair formation of the Nme1-Prune-1 complex within tumorigenic cells.

To address this issue, we developed CPP, which mimicks the minimal region of interaction of Nme-1 with Prune-1, from amino acids 115 to 129 , and thus it contains the crucial residues that can be phosphorylated by CKI (i.e., S120, S122, and S125). CPP has been shown to impair the Nme1-Prune-1 interaction complex in several tumorigenic cell lines. As a consequence of this impaired Nme-1-Prune-1 interaction, CPP reduced cell proliferation and cell motility across a panel of solid tumor cell lines, thus representing a novel strategy to inhibit tumor progression (Fig. 2b). These anti-tumorigenic effects have been shown in vitro in breast, prostate and colorectal tumors, and in neuroblastoma. The expression of CPP in these tumorigenic cells was achieved by infection with adenoviral particles carrying the sequence that encodes CPP fused to the transactivating protein from human immunodeficiency virus. The same approach was used to investigate the therapeutic effects of CPP in vivo in xenograft models of prostate and neuroblastoma tumors. In these in-vivo trials, CPP decreased the metastatic dissemination following tumor implantation in nude mice, thus indicating the potential for application of CPP to inhibition of metastasis formation in prostate cancer and neuroblastoma.

Of interest, CPP did not show toxic effects in vitro, as it did not affect cell proliferation or induce caspase-3-induced cell apoptosis in non-tumorigenic cells (i.e., HEK-293T, mouse embryonic fibroblasts [11], thus suggesting its specific action against tumorigenic cells. Most importantly, CPP was administered in the same way in vivo in a metastasis assay of the orthotopic murine model of metastatic prostate cancer (i.e., PC3 cell lines stably expressing firefly luciferase activity). The treated mice did not show any side effects in terms of haematological parameters, thus showing the biosafety of CPP also in vivo. However, a more safe and effective delivery system (e.g., using nanoliposomes) might overcome the problems of the adenovirusbased delivery system. Future studies that investigate the efficacy of a nanoparticle-CPP delivery system could address novel cancer therapeutic strategies for tumors requiring ectopic administration (e.g., brain tumors).

However, as CPP is a mimetic peptide of a phosphorylated domain of Nme-1, it might impair the interactions of Nme-1 with several other proteins, and thus impair other biological functions in cells. Indeed, Nme-1 and Prune-1 have both been described as 'sticky' proteins, and the identification of other protein interactors would be useful to better understand their biochemical functions and their involvement in tumor metastasis processes. Future studies will be aimed at gaining deeper insights into the mechanisms of proliferation and migration inhibition mediated by the CPP action in modulating the protein networks responsible for switching to pathological conditions [70, 36].

Furthermore, in treated tumorigenic cells, CPP also impaired the AKT/ mTOR and NF-kB pathways, thus also suggesting a role for the Nme-1-Prune-1 protein complex in these signaling cascades. Interestingly, both of these pathways are involved in the functions of inorganic polyphosphates in cells, in terms of cell proliferation and inflammation [71, 72]. Along these lines, Nme-1 was reported to inhibit the protein phosphatase enzymatic activity of Prune-1 [31]. Future studies will also be performed to determine the effects of the Nme-1-Prune-1 protein complex in terms of its role in the modulation of the levels of inorganic polyphosphates in neural cells, which have already 
been shown to regulate cell signaling through voltage-gated channels [73] . Furthermore, dissection of the role of inorganic polyphosphates in the mammalian nervous system would be of special interest, where both the Nme-1 and Prune- 1 proteins and their complex formation have potential roles during brain and cerebellum development [16].

Moreover, homozygous recessive mutations in Prune-1 have been shown for several families worldwide that are afflicted with microcephaly and PEHO-like syndrome [74, 75]. These gain-of-function mutations result in increased Prune-1 PPX/PPase activity in all of the mutant Prune-1 proteins, with delays in microtubule polymerization rates together with interactions with both $\beta$-tubulin and $\alpha$-tubulin on the mitotic spindle during mitosis, which thus affects the cell proliferation and migration processes [75]. Of interest, Nme-1 has also been reported to interact with microtubules [76] and its biochemical nucleotide diphospho kinase (NDPK) activity within centrosomes during cell division has also been described [77, 78]. Of note, IC261 inhibition of CKI $\delta / \varepsilon$, which are responsible for Nme-1 phosphorylation, is also known to impair microtuble depolymerization [56]. Altogether, these data suggest an additional role for the Nme-1-Prune-1 complex during cell division and mitosis, and so anomalies due to incorrect formation of the Nme-1-Prune-1 complex in affected children might result in neural developmental defects in the central nervous system (e.g., in brain, cerebellum, and optic nerve). To better dissect out these hypotheses, future studies need to investigate this potential "lethal interaction" in microcephaly and PEHO-like syndrome affected patients. This would thus open a novel scenario in which the therapeutic effects of CPP can also be investigated in these human genetic neurodevelopmental disorders.

To date, the presence of Nme-1 in the extracellular compartment "serum" from affected patients has not been deeply investigated in terms of its diagnostic potential. Here, extracellular Nme-1 and Prune-1 have been reported for serum of patients with breast cancer and lung cancer, respectively $[46,79]$. Together with impairment of NF-kB in CPP-treated tumorigenic cells [11], these findings suggest a role for the Nme-1-Prune-1 complex also in the inflammation cascade, and subsequently in modulation of the tumor microenvironment.

In summary, CPP has been shown to impair Nme1-Prune-1 protein complex formation and has demonstrated benefits in terms of cell proliferation and migration, induction of cell apoptosis, and inhibition of metastatic dissemination both in vitro and in vivo in different tumorigenic cells and in xenograft murine models (i.e., breast, prostate and colorectal cancers, neuroblastoma). These data represent a novel therapeutic option for the treatment of metastastatic dissemination in metastatic tumors that are characterized by alterations in Nme-1 and
Prune-1 protein levels. However, at this stage, these first analyzes of the therapeutic potential of CPP might only represent the "tip of the iceberg".

Acknowledgements We thank the following Resource Agencies for grant support: Fondazione Adolfo Volpe e Associazione Pediatri di Famiglia; EU-FP7-TUMIC-HEALTH-F2-2008-2016662 (MZ); the Italian Association for Cancer research (AIRC) Grant IG \# 11963 (MZ); the Regione Campania L.g.R: N.5 (MZ); the European National Funds PON01-02388/1 2007-2013 (MZ); POR Rete delle Biotecnologie in Campania Movie (MZ); and the European School of Molecular Medicine (SEMM) for a Fellowship (VF). The authors dedicate this study to the parents of Leonardo Andrini (an MB-group3-affected child), who died of cancer in October 2015.

\section{Compliance with ethical standards}

Conflict of interest The authors declare that they have no conflict of interest.

\section{References}

1. Stelzl U, Worm U, Lalowski M, et al. A human protein-protein interaction network: a resource for annotating the proteome. Cell. 2005;122:957-68.

2. Basse MJ, Betzi S, Bourgeas R, et al. 2P2Idb: a structural database dedicated to orthosteric modulation of protein-protein interactions. Nucleic Acids Res. 2013;41:D824-7.

3. Higueruelo AP, Schreyer A, Bickerton GR, Pitt WR, Groom CR, Blundell TL. Atomic interactions and profile of small molecules disrupting protein-protein interfaces: the TIMBAL database. Chem Biol Drug Des. 2009;74:457-67.

4. Heitz F, Morris MC, Fesquet D, Cavadore JC, Doree M, Divita G. Interactions of cyclins with cyclin-dependent kinases: a common interactive mechanism. Biochemistry. 1997;36:4995-5003.

5. Zhou X, Cao B, Lu H. Negative auto-regulators trap p53 in their web. J Mol Cell Biol. 2017;9:62-8.

6. Wu X, Bayle JH, Olson D, Levine AJ. The p53-mdm-2 autoregulatory feedback loop. Genes Dev. 1993;7:1126-32.

7. Green DR, Kroemer G. Cytoplasmic functions of the tumour suppressor p53. Nature. 2009;458:1127-30.

8. Vassilev LT, Vu BT, Graves B, et al. In vivo activation of the p53 pathway by small-molecule antagonists of MDM2. Science. 2004;303:844-8.

9. D'Angelo A, Garzia L, Andre A, et al. Prune cAMP phosphodiesterase binds nm23-H1 and promotes cancer metastasis. Cancer Cell. 2004;5:137-49.

10. Carotenuto M, Pedone E, Diana D, et al. Neuroblastoma tumorigenesis is regulated through the Nm23-H1/h-Prune C-terminal interaction. Sci Rep. 2013;3:1351.

11. Carotenuto M, de Antonellis P, Chiarolla CM, et al. A therapeutic approach to treat prostate cancer by targeting Nm23-H1/h-Prune interaction. Naunyn Schmiede Arch Pharmacol. 2015;388: 257-69.

12. Sturtevant AH. A highly specific complementary lethal system in Drosophila Melanogaster. Genetics. 1956;41:118-23.

13. Timmons L, Shearn A. Germline transformation using a prune cDNA rescues prune/killer of prune lethality and the prune eye color phenotype in Drosophila. Genetics. 1996;144:1589-600.

14. Reymond A, Volorio S, Merla G, et al. Evidence for interaction between human PRUNE and nm23-H1 NDPKinase. Oncogene. 1999;18:7244-52. 
15. Teng DH, Engele CM, Venkatesh TR. A product of the prune locus of Drosophila is similar to mammalian GTPase-activating protein. Nature. 1991;353:437-40.

16. Carotenuto P, Marino N, Bello AM, et al. PRUNE and NM23-M1 expression in embryonic and adult mouse brain. J Bioenerg Biomembr. 2006;38:233-46.

17. Bilitou A, De Marco N, Bello AM, et al. Spatial and temporal expressions of prune reveal a role in Muller gliogenesis during Xenopus retinal development. Gene. 2012;509:93-103.

18. Steeg PS, Bevilacqua G, Pozzatti R, Liotta LA, Sobel ME. Altered expression of NM23, a gene associated with low tumor metastatic potential, during adenovirus 2 Ela inhibition of experimental metastasis. Cancer Res. 1988;48:6550-4.

19. Urano T, Takamiya K, Furukawa K, Shiku H. Molecular cloning and functional expression of the second mouse nm23/NDP kinase gene, nm23-M2. FEBS Lett. 1992;309:358-62.

20. Rosengard AM, Krutzsch HC, Shearn A, et al. Reduced Nm23/ Awd protein in tumour metastasis and aberrant Drosophila development. Nature. 1989;342:177-80.

21. Leone A, Flatow U, King CR, et al. Reduced tumor incidence, metastatic potential, and cytokine responsiveness of nm23transfected melanoma cells. Cell. 1991;65:25-35.

22. Miyazaki H, Fukuda M, Ishijima Y, et al. Overexpression of $\mathrm{nm} 23-\mathrm{H} 2 / \mathrm{NDP}$ kinase B in a human oral squamous cell carcinoma cell line results in reduced metastasis, differentiated phenotype in the metastatic site, and growth factor-independent proliferative activity in culture. Clin Cancer Res. 1999;5:4301-7.

23. Lee JH, Cho SJ, Zhang X, et al. nm23-H1 protein expression and gene mutation in 150 patients with non-Hodgkin's lymphomas. J Korean Med Sci. 2006;21:645-51.

24. Oda Y, Naka T, Takeshita M, Iwamoto Y, Tsuneyoshi M. Comparison of histological changes and changes in $\mathrm{nm} 23$ and c-MET expression between primary and metastatic sites in osteosarcoma: a clinicopathologic and immunohistochemical study. Hum Pathol. 2000;31:709-16.

25. Niitsu N, Okamoto M, Okabe-Kado J, et al. Serum nm23-H1 protein as a prognostic factor for indolent non-Hodgkin's lymphoma. Leukemia. 2001;15:832-9.

26. Wallet V, Mutzel R, Troll $\mathrm{H}$, et al. Dictyostelium nucleoside diphosphate kinase highly homologous to Nm23 and Awd proteins involved in mammalian tumor metastasis and Drosophila development. J Natl Cancer Inst. 1990;82:1199-202.

27. Fuhs SR, Meisenhelder J, Aslanian A, et al. Monoclonal 1- and 3phosphohistidine antibodies: new tools to study histidine phosphorylation. Cell. 2015;162:198-210.

28. Wagner PD, $\mathrm{Vu}$ ND. Histidine to aspartate phosphotransferase activity of nm23 proteins: phosphorylation of aldolase C on Asp319. Biochem J. 2000;346:623-30.

29. Zhang Q, McCorkle JR, Novak M, Yang M, Kaetzel DM. Metastasis suppressor function of NM23-H1 requires its 3'-5' exonuclease activity. Int J Cancer. 2011;128:40-50.

30. Steeg PS, Zollo M, Wieland T. A critical evaluation of biochemical activities reported for the nucleoside diphosphate kinase/ Nm23/Awd family proteins: opportunities and missteps in understanding their biological functions. Naunyn Schmiede Arch Pharmacol. 2011;384:331-9.

31. Tammenkoski M, Koivula K, Cusanelli E, et al. Human metastasis regulator protein $\mathrm{H}$-prune is a short-chain exopolyphosphatase. Biochemistry. 2008;47:9707-13.

32. Zollo M, Andre A, Cossu A, et al. Overexpression of h-prune in breast cancer is correlated with advanced disease status. Clin Cancer Res. 2005;11:199-205.

33. Oue N, Yoshida K, Noguchi T, Sentani K, Kikuchi A, Yasui W. Increased expression of h-prune is associated with tumor progression and poor survival in gastric cancer. Cancer Sci. 2007;98:1198-205.
34. Hashimoto M, Kobayashi T, Tashiro H, Arihiro K, Kikuchi A, Ohdan H. h-Prune is associated with poor prognosis and epithelial-mesenchymal transition in patients with colorectal liver metastases. Int J Cancer. 2016;139:812-23.

35. Garzia L, D'Angelo A, Amoresano A, et al. Phosphorylation of $\mathrm{nm} 23-\mathrm{H} 1$ by CKI induces its complex formation with h-prune and promotes cell motility. Oncogene. 2008;27:1853-64.

36. Galasso A, Zollo M. The Nm23-H1-h-Prune complex in cellular physiology: a 'tip of the iceberg' protein network perspective. Mol Cell Biochem. 2009;329:149-59.

37. D'Angelo A, Zollo M. Unraveling genes and pathways influenced by H-prune PDE overexpression: a model to study cellular motility. Cell Cycle. 2004;3:758-61.

38. Diana D, Smaldone G, De Antonellis P, et al. Mapping functional interaction sites of human prune C-terminal domain by NMR spectroscopy in human cell lysates. Chemistry. 2013;19: 12217-20.

39. Ubersax JA, Ferrell JE Jr. Mechanisms of specificity in protein phosphorylation. Nat Rev Mol Cell Biol. 2007;8:530-41.

40. Middelhaufe S, Garzia L, Ohndorf UM, Kachholz B, Zollo M, Steegborn C. Domain mapping on the human metastasis regulator protein h-Prune reveals a C-terminal dimerization domain. Biochem J. 2007;407:199-205.

41. Polyak K. Heterogeneity in breast cancer. J Clin Invest. 2011;121:3786-8.

42. Khan I, Steeg PS. Metastasis suppressors: functional pathways. Lab Invest. 2018;98:198-210.

43. Steeg PS. Metastasis suppressors alter the signal transduction of cancer cells. Nat Rev Cancer. 2003;3:55-63.

44. Sastre-Garau X, Lacombe ML, Jouve M, Veron M, Magdelenat H. Nucleoside diphosphate kinase/NM23 expression in breast cancer: lack of correlation with lymph-node metastasis. Int $\mathrm{J}$ Cancer. 1992;50:533-8.

45. Okabe-Kado J, Kasukabe T, Honma Y, Kobayashi H, Maseki N, Kaneko Y. Extracellular NM23 protein promotes the growth and survival of primary cultured human acute myelogenous leukemia cells. Cancer Sci. 2009;100:1885-94.

46. Yokdang N, Nordmeier S, Speirs K, Burkin HR, Buxton IL. Blockade of extracellular NM23 or its endothelial target slows breast cancer growth and metastasis. Integr Cancer Sci Ther. 2015;2:192-200.

47. Romani P, Papi A, Ignesti M, et al. Dynamin controls extracellular level of Awd/Nme1 metastasis suppressor protein. Naunyn Schmiede Arch Pharmacol. 2016;389:1171-82.

48. Rumjahn SM, Javed MA, Wong N, Law WE, Buxton IL. Purinergic regulation of angiogenesis by human breast carcinomasecreted nucleoside diphosphate kinase. $\mathrm{Br} \mathrm{J}$ Cancer. 2007;97:1372-80.

49. Yokdang N, Tellez JD, Tian H, et al. A role for nucleotides in support of breast cancer angiogenesis: heterologous receptor signalling. Br J Cancer. 2011;104:1628-40.

50. Hirai M, Yoshida S, Kashiwagi H, et al. 1q23 gain is associated with progressive neuroblastoma resistant to aggressive treatment. Genes Chromosomes Cancer. 1999;25:261-9.

51. Tarkkanen M, Kiuru-Kuhlefelt S, Blomqvist C, et al. Clinical correlations of genetic changes by comparative genomic hybridization in Ewing sarcoma and related tumors. Cancer Genet Cytogenet. 1999;114:35-41.

52. Tirkkonen M, Tanner M, Karhu R, Kallioniemi A, Isola J, Kallioniemi OP. Molecular cytogenetics of primary breast cancer by CGH. Genes Chromosomes Cancer. 1998;21:177-84.

53. Kudoh K, Ramanna M, Ravatn R, et al. Monitoring the expression profiles of doxorubicin-induced and doxorubicin-resistant cancer cells by cDNA microarray. Cancer Res. 2000;60:4161-6.

54. Forus A, D'Angelo A, Henriksen J, et al. Amplification and overexpression of PRUNE in human sarcomas and breast 
carcinomas-a possible mechanism for altering the nm23-H1 activity. Oncogene. 2001;20:6881-90.

55. Mashhoon N, DeMaggio AJ, Tereshko V, et al. Crystal structure of a conformation-selective casein kinase-1 inhibitor. J Biol Chem. 2000;275:20052-60.

56. Stoter M, Kruger M, Banting G, Henne-Bruns D, Knippschild U. Microtubules depolymerization caused by the CK1 inhibitor IC261 may be not mediated by CK1 blockage. PLoS One. 2014;9: e100090.

57. Miller KD, Siegel RL, Lin CC, et al. Cancer treatment and survivorship statistics, 2016. Cancer J Clin. 2016;66:271-89.

58. Igawa M, Rukstalis DB, Tanabe T, Chodak GW. High levels of $\mathrm{nm} 23$ expression are related to cell proliferation in human prostate cancer. Cancer Res. 1994;54:1313-8.

59. Andolfo I, De Martino D, Liguori L, et al. Correlation of NM23H1 cytoplasmic expression with metastatic stage in human prostate cancer tissue. Naunyn Schmiede Arch Pharmacol. 2011;384: 489-98.

60. Saffran DC, Raitano AB, Hubert RS, Witte ON, Reiter RE, Jakobovits A. Anti-PSCA mAbs inhibit tumor growth and metastasis formation and prolong the survival of mice bearing human prostate cancer xenografts. Proc Natl Acad Sci USA. 2001;98:2658-63.

61. Ferlay J, Soerjomataram I, Dikshit R, et al. Cancer incidence and mortality worldwide: sources, methods and major patterns in GLOBOCAN 2012. Int J Cancer. 2015;136:E359-86.

62. Oliveira LA, Artigiani-Neto R, Waisberg DR, Fernandes LC, Lima Fde O, Waisberg J. NM23 protein expression in colorectal carcinoma using TMA (tissue microarray): association with metastases and survival. Arq Gastroenterol. 2010;47:361-7.

63. Suzuki E, Ota T, Tsukuda K, et al. nm23-H1 reduces in vitro cell migration and the liver metastatic potential of colon cancer cells by regulating myosin light chain phosphorylation. Int $\mathrm{J}$ Cancer. 2004;108:207-11.

64. Esposito MR, Aveic S, Seydel A, Tonini GP. Neuroblastoma treatment in the post-genomic era. J Biomed Sci. 2017;24:14.

65. Tan CY, Chang CL. NDPKA is not just a metastasis suppressorbe aware of its metastasis-promoting role in neuroblastoma. Lab Invest. 2018;98:219-27.

66. Almgren MA, Henriksson KC, Fujimoto J, Chang CL. Nucleoside diphosphate kinase $\mathrm{A} / \mathrm{nm} 23-\mathrm{H} 1$ promotes metastasis of NB69derived human neuroblastoma. Mol Cancer Res. 2004;2:387-94.
67. Okabe-Kado J, Kasukabe T, Honma Y, Hanada R, Nakagawara A, Kaneko Y. Clinical significance of serum NM23-H1 protein in neuroblastoma. Cancer Sci. 2005;96:653-60.

68. Biggs J, Tripoulas N, Hersperger E, Dearolf C, Shearn A. Analysis of the lethal interaction between the prune and Killer of prune mutations of Drosophila. Genes Dev. 1988;2:1333-43.

69. Carotenuto M, de Antonellis P, Chiarolla CM, et al. A therapeutic approach to treat prostate cancer by targeting Nm23-H1/h-Prune interaction. Naunyn Schmiede Arch Pharmacol. 2015;388:257-69.

70. Garzia L, Roma C, Tata N, Pagnozzi D, Pucci P, Zollo M. Hprune-nm23-H1 protein complex and correlation to pathways in cancer metastasis. J Bioenerg Biomembr. 2006;38:205-13.

71. Wang L, Fraley CD, Faridi J, Kornberg A, Roth RA. Inorganic polyphosphate stimulates mammalian TOR, a kinase involved in the proliferation of mammary cancer cells. Proc Natl Acad Sci USA. 2003;100:11249-54.

72. Hassanian SM, Ardeshirylajimi A, Dinarvand P, Rezaie AR. Inorganic polyphosphate promotes cyclin D1 synthesis through activation of $\mathrm{mTOR} / \mathrm{Wnt} / \mathrm{beta}$-catenin signaling in endothelial cells. J Thromb Haemost;2016;14:2261-73.

73. Holmstrom KM, Marina N, Baev AY, Wood NW, Gourine AV, Abramov AY. Signalling properties of inorganic polyphosphate in the mammalian brain. Nat Commun. 2013;4:1362.

74. Salpietro V, Zollo M, Vandrovcova J, et al. The phenotypic and molecular spectrum of PEHO syndrome and PEHO-like disorders. Brain. 2017;140:e49.

75. Zollo M, Ahmed M, Ferrucci V, et al. PRUNE is crucial for normal brain development and mutated in microcephaly with neurodevelopmental impairment. Brain. 2017;140:940-52.

76. Lombardi D, Sacchi A, D'Agostino G, Tibursi G. The association of the Nm23-M1 protein and beta-tubulin correlates with cell differentiation. Exp Cell Res. 1995;217:267-71.

77. Roymans D, Vissenberg K, De Jonghe C, et al. Identification of the tumor metastasis suppressor $\mathrm{Nm} 23-\mathrm{H} 1 / \mathrm{Nm} 23-\mathrm{R} 1$ as a constituent of the centrosome. Exp Cell Res. 2001;262:145-53.

78. Du J, Hannon GJ. The centrosomal kinase Aurora-A/STK15 interacts with a putative tumor suppressor NM23-H1. Nucleic Acids Res. 2002;30:5465-75.

79. Carotenuto M, De Antonellis P, Liguori L, et al. H-Prune through GSK-3beta interaction sustains canonical WNT/beta-catenin signaling enhancing cancer progression in NSCLC. Oncotarget. 2014:5:5736-49. 